\title{
Paired box 6 (PAX6) regulates glucose metabolism via proinsulin processing mediated by prohormone convertase 1/3 (PC1/3)
}

\author{
J. H. Wen • Y. Y. Chen $\cdot$ S. J. Song $\cdot$ J. Ding $\cdot$ Y. Gao $•$ \\ Q. K. Hu • R. P. Feng • Y. Z. Liu • G. C. Ren • \\ C. Y. Zhang $\cdot$ T. P. Hong $\cdot$ X. Gao $\cdot$ L. S. Li
}

Received: 25 July 2008 / Accepted: 20 August 2008 / Published online: 26 November 2008

(C) Springer-Verlag 2008

\begin{abstract}
Aims/hypothesis Human patients with aniridia caused by heterozygous $P A X 6$ mutations display abnormal glucose metabolism, but the underlying molecular mechanism is largely unknown. Disturbed islet architecture has been proposed as the reason why mice with complete inactivation of paired box 6 (PAX6) in the pancreas develop diabetes. This is not, however, the case in human aniridia patients with heterozygous PAX6 deficiency and no apparent defects in pancreatic development. We investigated the molecular mechanism underlying the development of abnormal glucose metabolism in these patients.

Methods A human aniridia pedigree with a PAX6 R240Stop mutation was examined for abnormal glucose metabolism using an OGTT. The underlying mechanism
\end{abstract}

Electronic supplementary material The online version of this article (doi:10.1007/s00125-008-1210-x) contains supplementary material, which is available to authorised users.

J. H. Wen, Y. Y. Chen and S. J. Song contributed equally to this study.

J. H. Wen · Y. Y. Chen · S. J. Song • Q. K. Hu • R. P. Feng •

T. P. Hong $\cdot$ L. S. Li

Peking University Stem Cell Research Center,

China-Australian Center of Excellence for Stem Cell Sciences,

Beijing, People's Republic of China

J. Ding $\cdot$ Y. Gao $\cdot$ X. Gao $(\bowtie)$

Model Animal Research Center and State Key

Laboratory of Pharmaceutical Biotechnology, Nanjing University,

Xuefu Road, Pukou High-tech Zone,

Nanjing, Jiangsu 210093, People's Republic of China

e-mail: gaox@nicemice.cn

C. Y. Zhang

School of Life Sciences, Nanjing University,

Nanjing, Jiangsu, People's Republic of China was further investigated using Pax6 R266Stop mutant small-eye mice, which also have abnormal glucose metabolism similar to that in PAX6 R240Stop mutation human aniridia patients.

Results Paired box 6 (PAX6) deficiency, both in aniridia patients with a heterozygous PAX6 R240Stop mutation and in mice with a heterozygous Pax6 R266Stop mutation, causes defective proinsulin processing and abnormal glucose metabolism. PAX6 can bind to the promoter and directly upregulate production of prohormone convertase (PC) $1 / 3$, an enzyme essential for conversion of proinsulin to insulin. Pax6 mutations lead to $\mathrm{PC} 1 / 3$ deficiency, resulting in defective proinsulin processing and abnormal glucose metabolism.

Conclusions/interpretation This study indicates a novel function for PAX6 in the regulation of proinsulin processing and glucose metabolism via modulation of $\mathrm{PC} 1 / 3$

\author{
Y. Z. Liu • G. C. Ren \\ Chaoyang Central Hospital Eye Center, \\ Chaoyang, Liaoning, People's Republic of China \\ Y. Y. Chen · T. P. Hong $(\bowtie)$ \\ Department of Endocrinology and Metabolism, \\ Peking University Third Hospital, \\ 49 North Garden Road, Haidian, \\ Beijing 100191, People's Republic of China \\ e-mail: tpho66@bjmu.edu.cn \\ J. H. Wen • S. J. Song • Q. K. Hu • R. P. Feng $・$ L. S. Li $(\square)$ \\ Department of Cell Biology, \\ Peking University Health Science Center, \\ 38 Xueyuan Road, Haidian, \\ Beijing 100191, People's Republic of China \\ e-mail: lingsongli@hsc.pku.edu.cn
}


production. It also provides an insight into the abnormal glucose metabolism caused by heterozygous PAX6 mutations in humans and mice.

Keywords Glucose metabolism · PAX6 · PC1/3 . Proinsulin processing

$\begin{array}{ll}\text { Abbreviations } \\ \text { ACTH } & \text { Adrenocorticotropic hormone } \\ \text { ChIP } & \text { Chromatin immunoprecipitation } \\ \text { EIA } & \text { Electroimmunoassay } \\ \text { GLP-1 } & \text { Glucagon-like peptide-1 } \\ \text { IGT } & \text { Impaired glucose tolerance } \\ \text { IPGTT } & \text { Intraperitoneal glucose tolerance test } \\ \alpha-M S H & \text { Alpha-melanocyte stimulating hormone } \\ \text { PAX6 } & \text { Paired box 6 } \\ \text { PC } & \text { Prohormone convertase } \\ \text { POMC } & \text { Pro-opiomelanocortin } \\ \text { qRT-PCR } & \text { Quantitative reverse transcription PCR } \\ \text { SSCP } & \text { Single-strand conformational polymorphism }\end{array}$

\section{Introduction}

PAX6 encodes a transcription factor and belongs to the paired-box gene family. The protein contains a paired domain, a linker region, a homeodomain and a transactivation region $[1,2]$. The paired domain together with the homeodomain binds to the promoter DNA, allowing the transactivation region to regulate the expression of its target gene $[3,4]$.

Paired box 6 (PAX6) is an evolutionarily conserved protein whose amino acid sequences are almost identical between humans and mice [5]. The Pax6-null mutant mouse has defects in the development of the eye, brain and pancreas, leading to neonatal lethality [6-10]. Mice with heterozygous Pax6 mutations can survive but show a phenotype of small eyes [8]. This small-eye phenotype mirrors the condition seen in human aniridia patients, who are characterised by lack of irises caused by heterozygous PAX6 gene mutations $[9,10]$.

In 2002, it was reported that four sporadic aniridia patients with $P A X 6$ mutations developed impaired glucose tolerance (IGT) [11], but the mechanism of how PAX6 heterozygosity leads to abnormal glucose metabolism is still unclear. Another study showed that mice with a complete loss of PAX6 in the pancreas developed diabetes [12]; these mutant mice exhibited disturbed islet architecture, suggesting that diabetes in Pax6 mutant mice is due to abnormal pancreatic development [7, 12, 13]. This, however, is not the case in heterozygous Pax6 mutant animals, which do not show apparent defects in pancreatic development [14]. Therefore, it is still largely unknown why aniridia patients with PAX6 mutations develop abnormal glucose metabolism.

To address these questions, we investigated an extended aniridia family and identified a pedigree carrying a heterozygous PAX6 gene mutation. We also obtained mutant mice that had both the Pax6 gene mutation and the small-eye phenotype. Using this heterozygous Pax6 mutant mouse model, we further investigated how the Pax6 gene mutation caused abnormal glucose metabolism in these mice and probably in the human aniridia patients, too.

\section{Methods}

Participants: identification of a pedigree with the PAX6 gene mutation The family with aniridia was identified at Peking University Third Hospital. This study was approved by the Ethics Committee of Peking University Health Science Center and carried out according to the guidelines of the World Medical Association Declaration of Helsinki (www.wma.net/e/policy/b3.htm). All participants (or their parents) were fully aware of the scope of this study and signed an informed consent form prior to enrolment.

The aniridia pedigree consisted of 19 living patients (Fig. 1a) and four unaffected consanguineous members (Fig. 1a). Of the 19 patients, 16 (all patients except for II-5, IV-10 and IV-11; Fig. 1a) were available for this study, together with all four unaffected consanguineous members (IV-3, IV-4, IV-6 and IV-8; Fig. 1a). To screen for the PAX6 mutation, each exon of the $P A X 6$ gene was amplified from genomic DNA as others have reported [9, 15]. The mutation site was determined by analysing PCR products from each exon by single-strand conformational polymorphism (SSCP) [16]. The PCR products found to have an abnormal pattern were sequenced to confirm the mutation.

Collection of human and mouse blood samples Venous blood samples were collected from all available members in the family at fasting and at 30,60 and $120 \mathrm{~min}$ after glucose loading. The amount of glucose ingested for adults was $75 \mathrm{~g}$ per person. For children this was adjusted according to the formula $1.75 \mathrm{~g} / \mathrm{kg}$ body weight, with total intake held to less than $75 \mathrm{~g}$. Genomic DNA was prepared from venous blood samples.

For the intraperitoneal glucose tolerance test (IPGTT) in mice, the blood samples were collected at fasting and at 30, 60 and 120 min after injection of glucose. Blood samples were collected from the tail vein into tubes containing anticoagulant (about 1,000 U of heparin per millilitre of blood). Immediately after centrifugation $(2,500 \mathrm{~g})$ at $4^{\circ} \mathrm{C}$, plasma was separated and stored at $-70^{\circ} \mathrm{C}$ for further analysis. 


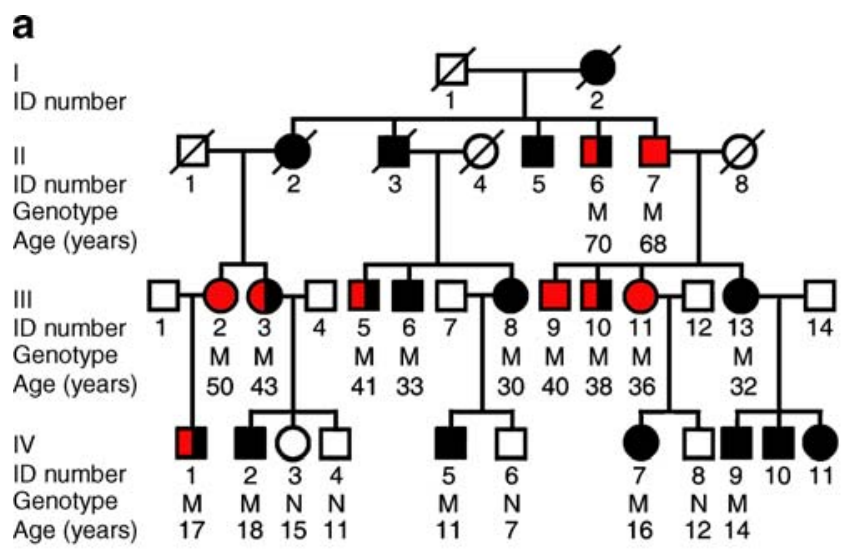

b

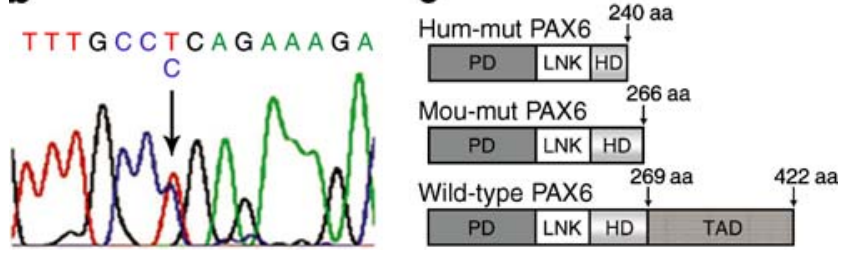

Fig. 1 PAX6 gene mutation leads to IGT and diabetes. a Illustration of a single human pedigree with the heterozygous PAX6 mutation, in which nine of 16 available patients developed IGT or diabetes. White symbols, family members without PAX6 mutation; black symbols, participants with the PAX6 mutation but without abnormal glucose metabolism; red symbols, patients with diabetes; black and red symbols, patients with IGT. N, normal allele; M, mutant allele. Genotype and age (years) of each participant are indicated under the ID number. b Sequence of the PCR product of exon 9 in PAX6. Arrow indicates the mutation site. A heterozygous $\mathrm{C}$ to $\mathrm{T}$ mutation converts an arginine codon (CGA) to a stop codon (TGA). c Schematic mapping of the wild-type PAX6, human mutant PAX6 (Hum-mut PAX6) and mouse mutant PAX6 (Mou-mut PAX6). The PAX6 polypeptide consists of paired domain (PD), linker region (LNK), homeodomain (HD) and transactivation domain (TAD). aa, amino acid

Measurement of plasma total insulin, proinsulin, adrenocorticotropic hormone and $\alpha$-melanocyte stimulating hormone Human insulin and proinsulin levels were measured with RIA kits (Linco Research, St Charles, MO, USA). Specific electroimmunoassay (EIA) kits from Phoenix Pharmaceuticals (Mountain View, CA, USA) were used to detect adrenocorticotropic hormone (ACTH) and alphamelanocyte stimulating hormone $(\alpha-\mathrm{MSH})$ levels in humans. The American Diabetes Association criteria were used for diagnosis of IGT and diabetes [17, 18].

Mouse insulin was measured with an ELISA kit from Shibayagi Company (Ishihara Shibukawa-shi, Gumma, Japan). Mouse proinsulin was measured using an ELISA kit for rat proinsulin (Mercodia, Uppsala, Sweden) according to the manufacturer's instructions. Since this kit is for rat proinsulin, the calculated ratios of proinsulin over total insulin in mice are likely to be not precise, but are acceptable for the present purposes of comparison. Specific
EIA kits and protocols (Phoenix Pharmaceuticals, Mountain View, CA, USA) were used to measure levels of ACTH and $\alpha$-MSH in tissue extracts of mice.

Animals, $N$-ethyl-N-nitrosourea mutagenesis, mapping and genotyping $\mathrm{C} 57 \mathrm{BL} / 6 \mathrm{~J}$ and $\mathrm{C} 3 \mathrm{H} / \mathrm{HeJ}$ mice were obtained from the Jackson Laboratory (Bar Harbor, ME, USA). Injection of the chemical mutagen $N$-ethyl- $N$-nitrosourea into male mice often induces sporadic A-T transition in the germ cell genome of the animals. After breeding with normal mice, the mutation can be transmitted to newborns. Based on the small-eye phenotype, mice with the Pax 6 gene mutation can be selected. The mutant mice were crossed to $\mathrm{C} 3 \mathrm{H} / \mathrm{HeJ}$ to generate F1 mice. The F1 mice with the small-eye phenotype were back-crossed to $\mathrm{C} 57 \mathrm{BL} / 6 \mathrm{~J}$ to generate $\mathrm{F} 2$. Tail DNA samples of F2 mice were extracted and PCRamplified with D2Mit249 micro-satellite marker of the Whitehead/MIT database (http://www-genome.wi.mit.edu). PCR products were separated by electrophoresis with $4 \%$ (wt/vol.) agarose II gel (BioBasic, Toronto, ON, Canada). Mice were genotyped by PCR analysis with primers specific for Pax6: forward primer 5'-GATGAGATGGGAGTTGGGTG-3', reverse primer: 5'-AAGTTACCAGGTGTGGTGGG-3'.

Animal care Mice were maintained in a pathogen-free facility under a $12 \mathrm{~h}$ light-dark cycle and were given free access to food and water. The studies were approved by the Peking University animal ethics committee. Animal experiments were carried out in accordance with institutional guidelines and complied with The 1996 Guide for the Care and Use of Laboratory Animals [19].

IPGTT and insulin tolerance test in mice After fasting for about $15 \mathrm{~h}$, baseline blood glucose levels were measured. The glucose solution $(20 \%$, wt $/ v o l$.) was then injected intraperitoneally at $10 \mu \mathrm{l} / \mathrm{g}$ body weight and blood glucose levels were measured at 30,60 and $120 \mathrm{~min}$ after the injection. For the insulin tolerance test, mice were fasted for $6 \mathrm{~h}$ and then injected with human insulin (Novo-Nordisk, Bagsværd, Denmark) at $0.75 \mathrm{U} / \mathrm{kg}$ body weight. Blood glucose levels were measured at fasting and at 15, 30, 45 and $60 \mathrm{~min}$ following insulin injections. Blood glucose concentrations were measured with a One Touch Ultra meter (Lifescan, Milpitas, CA, USA).

Isolation and purification of mouse islets The islets of mice were isolated and characterised following the method of Hori [20]. Briefly, the pancreas was digested by type V collagenase (Sigma-Aldrich, St Louis, MO, USA) and then purified by centrifugation $(2,700 \mathrm{~g})$ on Histopaque-1077 gradients (Sigma-Aldrich). Individual islets, free of attached acinar, vascular and ductal tissues, were handpicked under a dissecting microscope. 
Immunofluorescence The mouse pancreas was fixed for $10 \mathrm{~h}$ in $4 \%$ (wt/vol.) paraformaldehyde/PBS at $4^{\circ} \mathrm{C}$ before cryosection. The cryosections were immunostained with normal rabbit or mouse IgG, rabbit antibody to glucagon or mouse antibody to insulin (Zhongshan Goldenbridge Biotechnology, Beijing, China) and incubated overnight at $4^{\circ} \mathrm{C}$ with anti-rabbit $\operatorname{IgG}$ or anti-mouse $\operatorname{IgG}$, and then with mounting medium (DAPI; Vector Laboratories, Burlingame, CA, USA).

Cell culture NIT-1 mouse insulinoma cells were cultured in RPMI 1640 with $10 \%$ (vol./vol.) FBS. NIH3T3 cells were cultured in DMEM/High Glucose with $10 \%$ (vol./vol.) FBS. All cell culture media were obtained from Gibco BRL (Gaithersburg, MD, USA) and the FBS from Hyclone (Thermo Fisher Scientific, Waltham, MA, USA).

Quantitative RT-PCR RNA of islets was extracted with RNeasy Micro Kit (Qiagen, Valencia, CA, USA) and reversed to cDNA. The quantitative RT-PCR (qRT-PCR) was performed with primers specific for $P c 1 / 3$ (also known as Pcsk1): forward primer 5'-ATGGGCGGCGGAGATC-3', reverse primer 5'-CCAATCTGACCCAAAAGGTCATAC-3'. $G A P D H$ was used as a control: forward primer 5'-GCACC ACCAACTGCTTAGC-3', reverse primer 5'-TCTTCTGGG TGGCAGTGATG-3'.

Western blotting analysis The proteins of islets were separated by $10 \%$ (wt/vol.) SDS-PAGE and probed with different antibodies including rabbit PAX6-specific antibody (Chemicon, Temecula, CA, USA), rabbit prohormone convertase (PC)1/3specific antibody (Abcam, Cambridge, MA, USA) and mouse $\beta$-actin-specific monoclonal antibody (Sigma-Aldrich).

Luciferase reporter assay A 4,194-bp mouse Pc1/3 promoter sequence was cloned from mouse genomic DNA and inserted into $\mathrm{pGL}_{3}$-basic luciferase reporter vectors. The primers were as follows: forward primer 5'-CGGGGTACC GGGCTGGGAAGGAATAAAATCTGGAGTATAAA-3', reverse primer 5'-CCGCTCGAGTCTTGACGTCAGATC TACCTGGACTGAGAACTGGAT-3'. Pax6 was cloned and inserted into pcDNA3.1 (-) vectors. The luciferase reporter assay was performed in NIH3T3 cells. The cells were cultivated in 24 well plates for $24 \mathrm{~h}$ and transfected by transfection reagent (Lipofectamine 2000; Invitrogen, Carlsbad, CA, USA) with the mouse $P c 1 / 3$ promoter along with either intact or mutated Pax6 cDNA at several doses. Cells were collected $48 \mathrm{~h}$ after transfection and assayed in triplicate with the Dual-Luciferase Reporter Assay System (Promega, Madison, WI, USA).

Chromatin immunoprecipitation assays Chromatin immunoprecipitation (ChIP) assays were performed in a mouse beta cell line, NIT-1 cells from the American Type Culture Collection (ATCC, Manassas, VA, USA). Briefly, the chemical-linked complexes of genomic DNA and PAX6 protein were immunoprecipitated with antibodies specific to PAX6 (Chemicon). The DNA was amplified after ChIP using primers of $\mathrm{Pcl} / 3$ promoter (forward primer $5{ }^{\prime}$-TCAT CAAGTCTTGCCTCGTT-3', reverse primer 5'-ATTAT CGCTGTCCACACAT-3').

Statistical analysis Statistical analysis was performed using an independent samples $t$ test. Differences were considered statistically significance at $p<0.05$.

\section{Results}

A heterozygous PAX6 R240Stop mutation was identified in the aniridia pedigree; affected patients exhibited abnormal proinsulin processing and impaired glucose tolerance To determine whether there was any mutation in the PAX6 gene in the identified pedigree, we first narrowed down the region bearing a potential mutation to exon 9 of the $P A X 6$ gene by SSCP (not shown). Within exon 9 we identified a C-to-T mutation at nucleotide 1080 that converted an Arg codon (CGA) to a stop codon (TGA; Fig. 1b). This mutation resulted in a truncated polypeptide that prematurely terminated at residue 240 (PAX6 R240Stop mutation; hereafter referred to as the PAX6 mutation; Fig. 1c), whereas the full length PAX6 consists of 422 amino acids (Fig. 1c). The R240Stop truncated polypeptide lacked the entire transactivation domain and a small portion of the homeodomain (Fig. 1c). The mutation was not found in the four unaffected consanguineous family members or in the sample of 100 unaffected people.

To investigate whether the stop mutation in PAX6 might lead to abnormal glucose metabolism in humans, we measured fasting glucose and insulin levels in the blood of each participant and then performed OGTT. Four nonmutation family members and 12 healthy adults were included as normal controls, as well as eight patients with type 2 diabetes (but without the PAX6 mutation, obesity or diabetic complications). While the patients with type 2 diabetes had significantly higher fasting glucose (Fig. 2a) and insulin levels (Fig. 2b) than the other two groups, there was no difference between the patients with the heterozygous PAX6 mutation and the normal controls (Fig. 2a,b). In OGTT, on the other hand, the post-load blood glucose levels in the patients with the PAX6 mutation were significantly higher than those in the controls (Fig. 2c). Interestingly, among the 19 available patients bearing the PAX6 mutation, seven aged 11 to 33 years (IV-5, IV-9, IV-7, IV-2, III-8, III-13, III-6) appeared to have normal glucose 

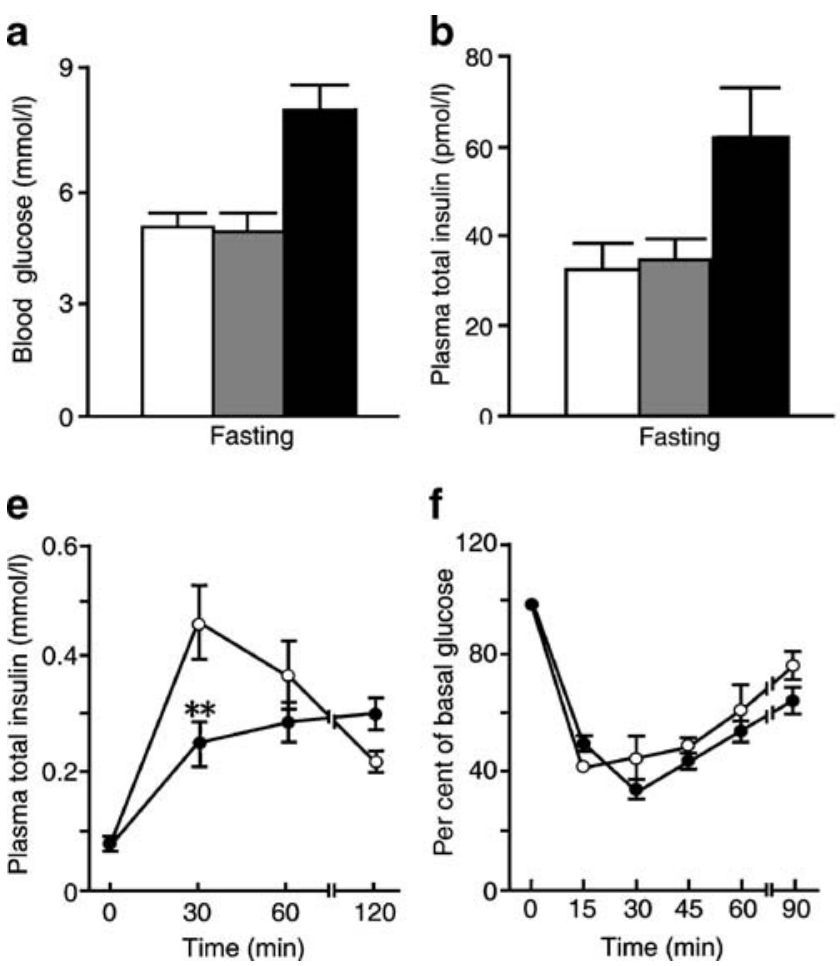

Fig. 2 Patients with the heterozygous $P A X 6$ mutation exhibit abnormal glucose metabolism. Fasting blood glucose levels (a) and plasma total insulin levels (b) in normal controls (white bars), patients with the PAX6 mutation (grey bars) and patients with diabetes (black bars). c Blood glucose levels in normal controls (white circles) and patients with the heterozygous $P A X 6$ mutation (black circles) as measured in OGTT. d The development of IGT (dashed line) and diabetes (continuous line) in patients with the heterozygous PAX6 mutation correlated with the ages of the patients. White triangles, normal controls; black triangles, patients with the PAX6 mutation. e Plasma total insulin levels in normal controls (white circles) and

metabolism despite having aniridia (Fig. 1a), but five aged 17 to 70 years (IV-1, III-10, III-5, III-3, II-6) had IGT (Fig. 1a) and four from ages 36 to 68 years (III-11, III-9, III-2, II-7) even developed diabetes (Fig. 1a). It appears that the phenotypes vary according to the ages of the patients bearing the PAX6 mutation (Fig. 2d). Neither non-mutation family members nor the 12 healthy adults exhibited abnormal glucose metabolism. The clinical variables are shown in Electronic supplementary material (ESM) Table 1.

Total insulin levels (including proinsulin and true insulin) in the blood of patients with the PAX6 mutation were significantly lower than those in the controls post glucose loading (Fig. 2e). At $2 \mathrm{~h}$ after the glucose challenge, total insulin production in the patients with the PAX6 mutation finally caught up with the levels in the controls (Fig. 2e); nonetheless, blood glucose levels in the same patients with the PAX6 mutation remained high (Fig. 2c). This suggests that these patients might have defects in insulin production and insulin signalling. To exclude the latter possibility, an insulin tolerance test was

\section{C}

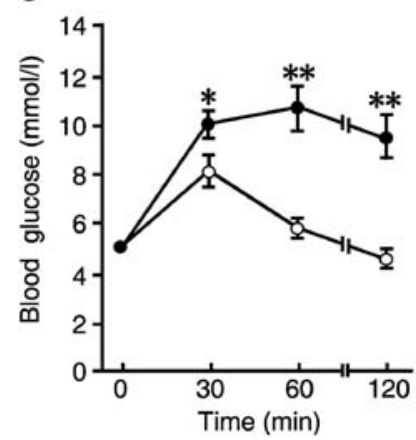

g

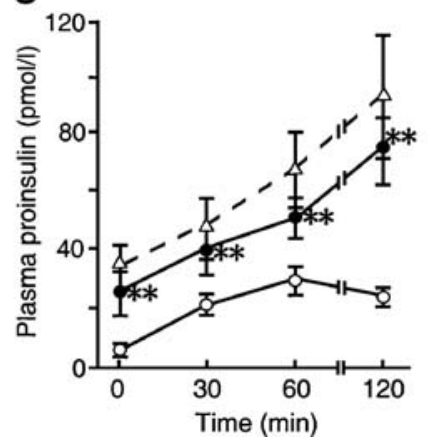

d

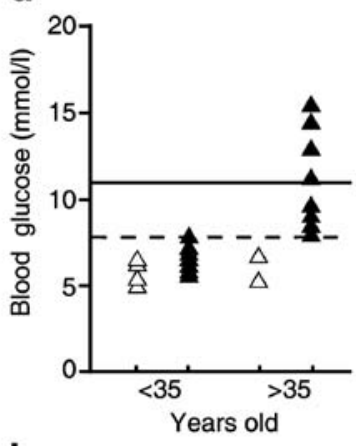

h

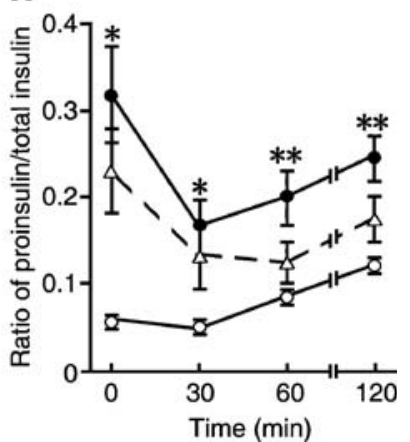

patients with the PAX6 mutation (black circles) during OGTT. f Insulin tolerance test. The percentage of blood glucose levels over basal levels at different time points after injection of insulin in normal controls (white circles) and in patients with the PAX6 mutation (black circles). g Plasma proinsulin levels in normal controls (white circles), patients with the PAX6 mutation (black circles) and in patients with diabetes (white triangles). $\mathbf{h}$ The plasma proinsulin/total insulin ratio in normal controls (white circles), patients with the PAX6 mutation (black circles) and patients with diabetes (white triangles). Each data point represents the mean \pm SE. ${ }^{*} p<0.05,{ }^{*} p p<0.01$

performed. The participants received an insulin injection and blood glucose levels were measured at different time points. As shown in Fig. $2 \mathrm{f}$, patients with the PAX6 mutation and controls showed no difference in terms of insulin sensitivity. Interestingly, the blood proinsulin levels (Fig. 2g) and the proinsulin/C-peptide ratios (ESM Fig. 1) were much higher in both diabetic patients and in the patients with the PAX6 mutation than in the controls.

Although blood proinsulin levels in the patients with the PAX6 mutation were lower than in the patients with type 2 diabetes (Fig. $2 \mathrm{~g}$ ), the proinsulin/total insulin ratio in the former was significantly higher than in the latter (Fig. 2h). Similar results were also observed when the patients with the PAX6 mutation were subdivided into groups with (1) normal glucose metabolism and (2) abnormal glucose metabolism, i.e. either IGT or diabetes (ESM Fig. 2). Therefore, unlike in the type 2 diabetes patients whose high proinsulin levels may be due to overstimulation by hyperglycaemia [21,22], the high proinsulin levels in the patients with the $P A X 6$ mutation most probably resulted from a 
defect in proinsulin processing. In other words, defective proinsulin processing may be the primary cause for development of abnormal glucose metabolism in patients with the PAX6 mutation.

Cleavage of proinsulin mainly depends on prohormone convertases. Both PC1/3 and PC2 are members of a family of serine proteases encoded on human chromosome 5q14q21and 20p11.2, respectively [23, 24]. While pancreatic alpha cells mainly produce PC2, pancreatic beta cells produce $\mathrm{PC} 1 / 3$ and $\mathrm{PC} 2$ [25-27]. Based on the pedigree study, we hypothesised that PAX6 may regulate the production of the $\mathrm{PCs}$ and that the PAX6 gene mutation causes PC deficiency, leading to defective proinsulin processing and, in turn, abnormal glucose metabolism.

Pax6 heterozygous R266Stop mutant mice exhibited abnormal glucose metabolism similar to that in the human patients with the PAX6 mutation To identify a Pax6 mutant mouse model that displays a mutation similar to the aniridia patients with the PAX6 mutation, we screened $N$-ethyl- $N$ nitrosourea mutagen-induced mutagenic mice for Pax6 gene mutations based on the typical small-eye phenotype of Pax6 mutants $[8,28]$ and identified a strain of mice with a truncation at residue 266 of the mouse PAX6 protein (Pax6 heterozygous R266Stop mutant mice; hereafter referred to as Pax6 mutant mice) (Figs 1c and 3a).

These mutant mice did not show obvious structural abnormalities in the pancreas (Fig. 3b) as revealed by immunofluorescent staining of insulin and glucagon. Glucose metabolism as measured by IPGTT appeared to be nearly normal in these mutant mice when younger than 4 months (Fig. 3c). However, when older than 6 months, most of these Pax 6 mutant mice, like patients with the PAX6 mutation above 35 years of age in the pedigree (Fig. 2d), exhibited IGT detected by IPGTT (Fig. 3c), but responded normally to exogenous insulin (Fig. 3d). Simi- a

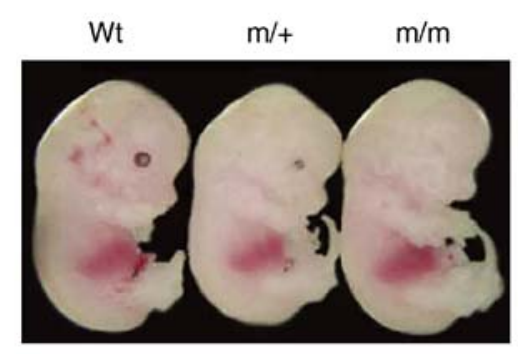

C

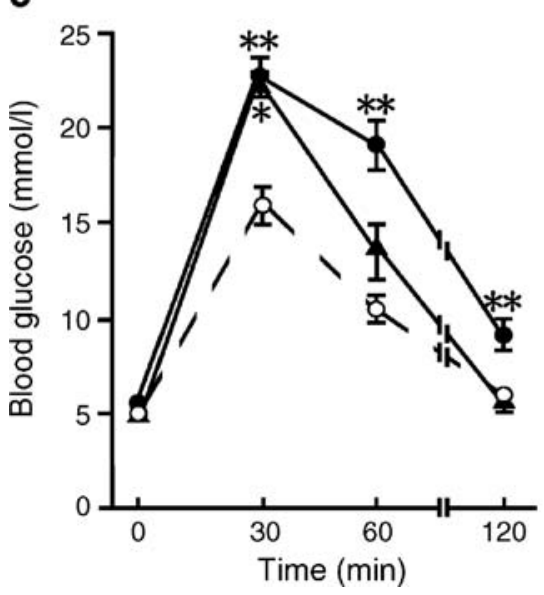

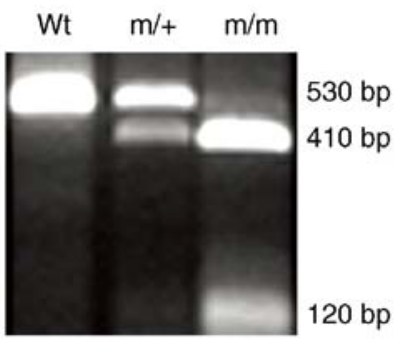

b
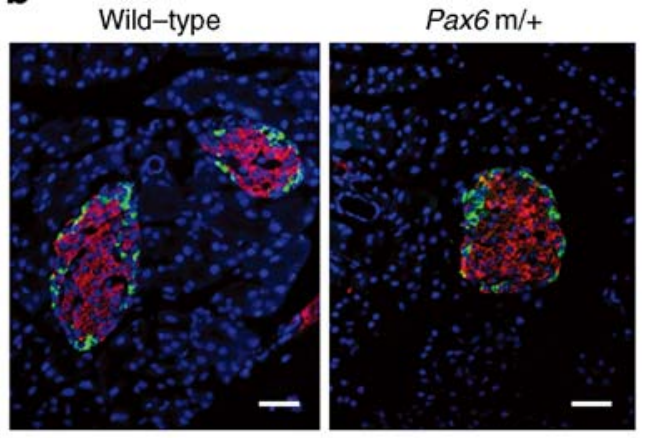

e

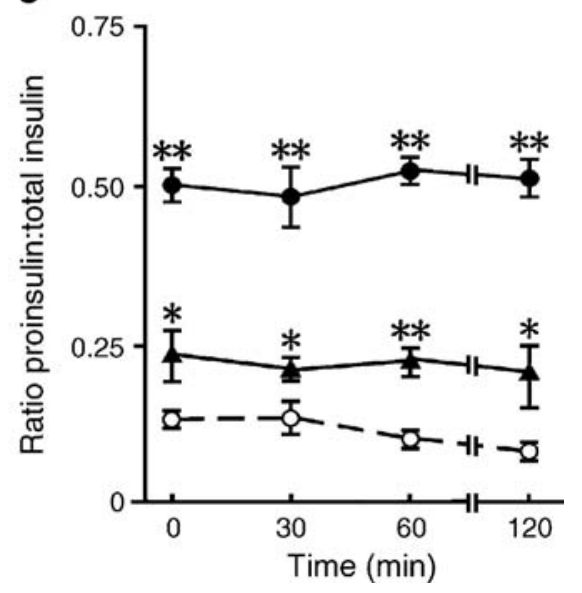

Fig. 3 Pax6 mutant mice (Pax6 $\mathrm{m} /+$ ) exhibited abnormal glucose metabolism similar to that observed in human patients with the heterozygous PAX6 mutation. a Photo showing phenotypes and genotypes for wild-type (Wt), Pax $6 \mathrm{~m} /+$ and Pax6 $\mathrm{m} / \mathrm{m}$ mice. b Immunofluorescent staining for total insulin and glucagon in the pancreas of these mice. Red, insulin; green, glucagon; blue, DAPI. Scale bar, $50 \mu \mathrm{m}$. c Blood glucose levels during IPGTT in wild-type mice $(n=18)$ (white circles) and in 4-month-old $(n=7)$ (black triangles) and 6-month-old (black circles) Pax6 m/+ mice $(n=13)$. d Insulin tolerance test. The percentage of blood glucose levels over the basal levels at different time points after injection of insulin in wild-type mice ( $n=6$; white circles) or in 6-month-old Pax $6 \mathrm{~m} /+$ mice ( $n=6$; black circles). e The plasma proinsulin/total insulin ratios in Pax6 mutant mice at ages 4 months $(n=7)$ (black triangles) or 6 months ( $n=6$; black circles) were significantly higher than those in wild-type mice ( $n=7$; white circles). Each value represents the mean \pm SE. ${ }^{*} p<0.05, * * p<0.01$ 
larly to the patients with the PAX6 mutation, the proinsulin/ total insulin ratios in the Pax6 mutant mice were significantly higher than in wild-type controls (Fig. 3e). Actually, a high proinsulin/total insulin ratio was also observed in mutant mice at age 4 months that had not shown abnormalities at $120 \mathrm{~min}$ in the IPGTT (Fig. 3c,e). These observations indicate that the abnormal proinsulin/total insulin ratio was present before the development of abnormal glucose metabolism in the Pax6 mutant mice.

In analysing glucose metabolism in these mutants, we documented that the Pax 6 mutant mice share a similarity with the human aniridia patients with the PAX6 mutation with regard to abnormal glucose metabolism. Using this animal model, we then investigated whether PCs are deficient and if so, how the Pax6 mutation leads to such a deficiency.

PAX6 directly regulates $P C 1 / 3$ levels and the Pax6 mutation leads to PC1/3 deficiency We first analysed the expression of $\mathrm{PC}$ genes in the Pax6 mutant mouse islets by qRT-PCR and found that, between $P c 1 / 3$ and $P c 2$, it was $P c 1 / 3$ that was significantly decreased in the islets of the Pax6 mutant mice (Fig. 4a). This decrease in Pcl/3 expression was then confirmed at the protein level by western blotting analysis (Fig. 4b, $\mathrm{PC} 1 / 3$ ).

a
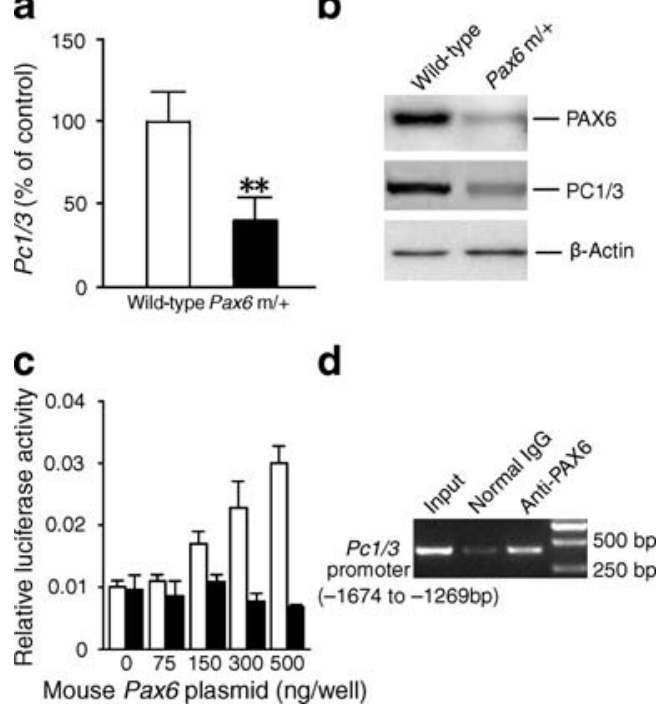

Fig. 4 PAX6 directly regulates $P c 1 / 3$ expression and Pax6 mutation leads to $\mathrm{PC} 1 / 3$ deficiency. Expression of $P c 1 / 3$ in isolated islets as measured by qRT-PCR (a) or by western blotting analysis (b) in wildtype versus Pax6 mutant mice. c PAX6 (white bars), but not the truncated PAX6 (black bars), upregulated $P c 1 / 3$ expression as analysed using a luciferase report system. d PAX6 binds directly to the $P c 1 / 3$ promoter in NIT- 1 cells as analysed by ChIP assay. Each bar represents the mean \pm SE. ${ }^{* *} p<0.01$
To investigate how the Pax6 mutation leads to PC1/3 deficiency, we performed a luciferase reporter assay using $P c 1 / 3$ promoter activity as the variable. PAX6, but not the truncated moiety, was found to promote the expression of $P c 1 / 3$ in a dose-dependent manner (Fig. 4c). In agreement with these data, we detected the binding of PAX6 to the $P c 1 / 3$ promoter by ChIP assay (Fig. 4d) in NIT-1 cells [29, 30]. We therefore concluded that the Pax6 mutation caused $\mathrm{PC} 1 / 3$ deficiency, leading to defective proinsulin processing. Since the truncated PAX6 polypeptide did not show any effect on $P c 1 / 3$ expression (Fig. 4c), we concluded that the Pax6 mutation leading to $\mathrm{PC} 1 / 3$ deficiency is a dosedependent phenomenon rather than a dominant negative effect.

ACTH and $\alpha-M S H$ were decreased in the blood of the aniridia patients with the PAX6 mutation as well as in the brain of Pax6 mutant mice In addition to the pancreas, $\mathrm{PC} 1 / 3$ is also present in the hypothalamus, where it converts pro-opiomelanocortin (POMC) into ACTH. ACTH is further converted into $\alpha-\mathrm{MSH}$, which plays a role in regulation of food intake in mice and humans [31]. If the PAX6 gene mutation does indeed decrease PC1/3 production, the production of ACTH as well as $\alpha$-MSH should be reduced in the brain. This was indeed the case. Both ACTH and $\alpha$-MSH were significantly decreased in the peripheral blood of the patients with the PAX6 mutation (Fig. 5a) as well as in the hypothalamus of the Pax6 mutant mice (Fig. 5b).

\section{Discussion}

Based on our investigation of aniridia patients with the heterozygous $P A X 6$ mutation and of small-eye mice with the Pax6 mutation, we demonstrated that the PAX6 mutation causes a decrease in $\mathrm{PC} 1 / 3$. The resulting $\mathrm{PC} 1 / 3$ deficiency leads to defective proinsulin processing and subsequent abnormal glucose metabolism. This study thus indicates a novel function for PAX6 in the regulation of proinsulin processing and glucose metabolism via modulation of $\mathrm{PC} 1 / 3$ production.

Our finding is consistent with studies by Yasuda et al. [11] and Ashery-Padan et al. [12] in that PAX6 deficiency leads to abnormal glucose metabolism in mice and in humans. Moreover, we provide evidence that PAX6 mutations cause $\mathrm{PC} 1 / 3$ deficiency, resulting in defective proinsulin processing and subsequent abnormal glucose metabolism. This conclusion is partially supported by the fact that $\mathrm{PC} 1 / 3$ deficiency causes abnormal glucose metabolism in humans and mice [32-35]. However, the phenotype and the primary mechanism may be different. 
a
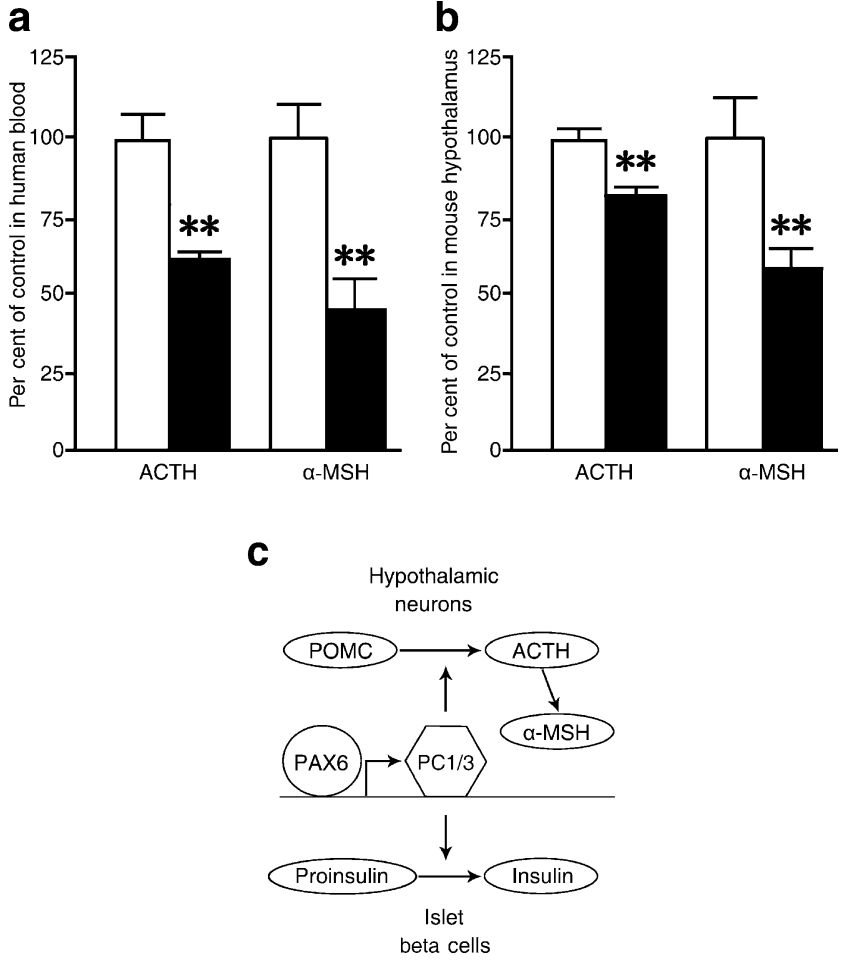

Fig. 5 ACTH and a-MSH levels were decreased in PAX6 haploinsufficiency mice and patients with heterozygous PAX6 mutations. a $\mathrm{ACTH}$ and $\alpha-\mathrm{MSH}$ peripheral blood contents was measured in humans by RIA. The plasma from patients with PAX6 mutations (black bars) and normal controls (white bars) was collected $120 \mathrm{~min}$ after oral glucose. Levels are shown as percentage of control levels (mean \pm SE). $n=6$ for Pax 6 mutant mice; $n=9$ for patients with PAX6 mutations. b ACTH and $\alpha$-MSH contents in hypothalamus tissue of mice were measured by RIA. Hypothalamus tissue of wild-type (white bars) and Pax6 mutant mice (black bars) was extracted 120 min after glucose ingestion to detect the concentration of ACTH and $\alpha$-MSH. c Proposed model for PAX6 in regulation of proinsulin processing in the islets and of POMC processing in the hypothalamus. Each bar represents the mean \pm SE. ${ }^{*} p<00.01$

For instance, human patients with $P C 1 / 3$ mutation or mice with $P c 1 / 3$ N222D/N222D mutation exhibited significant obesity $[33,35]$. In our study, we saw no obesity either in the Pax6 mutant mice or in the patients with the PAX6 mutation, as evidenced by their normal BMI (ESM Table 1), even though $\alpha$-MSH levels were decreased in the human and mice mutant groups. This observation is in relative agreement with what is seen in Pcl-null mice, which showed $\alpha$-MSH deficiency but did not develop obesity [34]. It appears that an obese phenotype such as this depends on the dosage of $\mathrm{PC} 1 / 3$, since mice with $\mathrm{PC} 1 / 3$ hemizygous deficiency indeed exhibited mild obesity [34].

It has been reported that $P a x 6^{\text {sey/sey }}$ mice with homozygous Pax6 mutation exhibited decreased levels of glucagon-like peptide-1 (GLP-1) in the small and large intestine [36]. However, we did not detect significant differences in GLP-1 production in the patients with the mutation versus normal controls (not shown). One possible explanation is that, unlike in mouse intestine, PAX6 may not be present in significant amounts in adult human intestinal cells, as suggested by a published study [37]. Compared with its production in human pancreas, $P A X 6$ expression was barely detected in human intestine [37]. Moreover, we found that all IVGTT measurements were very similar to those from the OGTT (ESM Fig. 3). These data indicate that the abnormal glucose metabolism we observed in this study cannot be due to changes in the intestinal GLP-1 level.

Since PAX6 controls many downstream events, the primary cause of abnormal glucose metabolism in the PAX6-deficient participants may differ from that in the PC1/3-deficient animals. Some of the downstream factors regulated by PAX6 may play a role opposed to that of $\alpha$-MSH in the regulation of food intake; even though both $\mathrm{PC} 1 / 3$ and $\alpha-\mathrm{MSH}$ were reduced in our participants, we did not observe obesity in those with the PAX6 mutation. The definitive explanation of these discrepancies awaits further investigation.

Using PAX6-deficient mouse models, several previous studies have proposed that the disturbed architecture and the resulting deficiency in insulin production in pancreas is the reason why mutant mice develop diabetes $[7,12,13]$. In heterozygous Pax6 mutant mice, however, we did not see any obviously abnormal pancreatic architecture (Fig. 3b) [14]. Instead, we found that the proinsulin/total insulin ratio both in the patients with the PAX6 mutation (Fig. 2g,h) and in the Pax6 mutant mice was significantly higher than in wild-type controls (Fig. 3e). Furthermore, we found that, even though type 2 diabetes patients often have high proinsulin levels due to overstimulation by hyperglycaemia $[21,22]$, the proinsulin/total insulin ratio in the patients with the heterozygous PAX6 mutation was significantly higher than in the type 2 diabetes patients (Fig. 2h), suggesting, again, that defective proinsulin processing may be the primary cause of diabetes in the patients with the PAX6 mutation. This observation of the effect of the PAX6 mutation is partially supported by a report that a point mutation in the human insulin gene gives rise to abnormal glucose metabolism and hyperproinsulinaemia [38].

It is important to note that the majority of the Pax6 mutant mice and the patients with the PAX6 mutation did not develop IGT until advanced ages. For example, most patients with the PAX6 mutation exhibited abnormal glucose metabolism at ages above 35 years (Fig. 2d), which was similar to the comparative age ( $>6$ months) at which abnormal glucose metabolism appeared in Pax6 mutant mice (Fig. 3c), suggesting that other age-dependent factors may possibly exist and work in coordination with PAX6 in the regulation of glucose metabolism. This also raises an interesting question regarding proinsulin levels in the participants with the heterozygous mutation in PAX6 who did not yet show abnormal glucose metabolism. 
Before age 4 months, the Pax 6 mutant mice did not show significant differences from wild-type mice in blood glucose levels during the IPGTT (Fig. 3c), but did exhibit higher proinsulin/total insulin ratio than wild-type animals (Fig. 3e). Therefore the high ratio of proinsulin/ total insulin in the Pax6 mutant mice occurs prior to onset of abnormal glucose metabolism, suggesting, again, defective proinsulin processing as the cause rather than an effect of abnormal glucose metabolism in the animals with the heterozygous Pax6 mutation. The presence of such additional factors that contribute to the development of abnormal glucose metabolism in the animals with the Pax6 mutation is currently under further investigation.

Glucose metabolism is an extraordinarily complex process that is regulated by multiple mechanisms. Our conclusion that defective proinsulin processing causes abnormal glucose metabolism in the participants with $P A X 6$ mutations does not exclude the possibility that other mechanisms, such as the $\alpha$-MSH regulatory pathway, may also contribute to the disorder. Nonetheless, our study provides a novel function of PAX6 in the regulation of proinsulin processing and glucose metabolism via modulation of $\mathrm{PC} 1 / 3$ production. It also provides an insight into how PAX6 mutations lead to abnormal glucose metabolism in mice and humans.

Acknowledgements We thank D. Stenger (Barbara Davis Center, University of Colorado, Denver, CO, USA) and L. H. Li (Stowers Institute for Medical Research, Kansas City, MO, USA) for their comments and suggestions. This work was supported by grants from the Chinese National 863 Projects (2006AA02A114), Beijing Ministry of Science and Technology (D07050701350704) and the Chinese National 973 Projects (2006CB943603) to L. S. Li, and the Chinese National 973 Projects (2006CB503905) to Peking University Stem Cell Research Center. It was also supported by grants from the Ministry of Science and Technology for International Collaboration Project 20070192 to the China-Australian Center of Excellence for Stem Cell Sciences, a grant from Natural Science Foundation of Beijing (7062067) to T. P. Hong and grants from the National Natural Science Foundation of China to J. H. Wen (30871251) and to C. Y. Zhang (Outstanding Young Scientist Award 30225037).

Duality of interest The authors declare that there is no duality of interest associated with this manuscript.

\section{References}

1. Mishra R, Gorlov IP, Chao LY, Singh S, Saunders GF (2002) PAX6, paired domain influences sequence recognition by the homeodomain. J Biol Chem 277:49488-49494

2. Mansouri A, Hallonet M, Gruss P (1996) Pax genes and their roles in cell differentiation and development. Curr Opin Cell Biol 8:851-857

3. Grzeskowiak R, Amin J, Oetjen E, Knepel W (2000) Insulin responsiveness of the glucagon gene conferred by interactions between proximal promoter and more distal enhancer-like elements involving the paired-domain transcription factor Pax6. J Biol Chem 275:30037-30045

4. Noll M (1993) Evolution and role of Pax genes. Curr Opin Genet Dev 3:595-605

5. Kozmik Z, Czerny T, Busslinger M (1997) Alternatively spliced insertions in the paired domain restrict the DNA sequence specificity of Pax6 and Pax8. EMBO J 16:6793-6803

6. Sisodiya SM, Free SL, Williamson KA et al (2001) PAX6 haploinsufficiency causes cerebral malformation and olfactory dysfunction in humans. Nat Genet 28:214-216

7. Sander M, Neubuser A, Kalamaras J, Ee HC, Martin GR, German MS (1997) Genetic analysis reveals that PAX6 is required for normal transcription of pancreatic hormone genes and islet development. Genes Dev 11:1662-1673

8. Quiring R, Walldorf U, Kloter U, Gehring WJ (1994) Homology of the eyeless gene of Drosophila to the small eye gene in mice and aniridia in humans. Science 265:785-789

9. Glaser T, Walton DS, Maas RL (1992) Genomic structure, evolutionary conservation and aniridia mutations in the human PAX6 gene. Nat Genet 2:232-239

10. Walther C, Gruss P (1991) Pax-6, a murine paired box gene, is expressed in the developing CNS. Development 113:14351449

11. Yasuda T, Kajimoto Y, Fujitani Y et al (2002) PAX6 mutation as a genetic factor common to aniridia and glucose intolerance. Diabetes 51:224-230

12. Ashery-Padan R, Zhou X, Marquardt T et al (2004) Conditional inactivation of Pax6 in the pancreas causes early onset of diabetes. Dev Biol 269:479-488

13. St-Onge L, Sosa-Pineda B, Chowdhury K, Mansouri A, Gruss P (1997) Pax6 is required for differentiation of glucagon-producing alpha-cells in mouse pancreas. Nature 387:406-409

14. Hamasaki A, Yamada Y, Kurose T et al (2007) Adult pancreatic islets require differential pax6 gene dosage. Biochem Biophys Res Commun 353:40-46

15. Love J, Axton R, Churchill A, van Heyningen V, Hanson I (1998) A new set of primers for mutation analysis of the human PAX6 gene. Human Mutat 12:128-134

16. Axton R, Hanson I, Danes S, Sellar G, van Heyningen V, Prosser $\mathrm{J}$ (1997) The incidence of PAX6 mutation in patients with simple aniridia: an evaluation of mutation detection in 12 cases. $\mathrm{J}$ Med Genet 34:279-286

17. No authors listed (2007) Standards of medical care in diabetes2007. Diabetes Care 30(Suppl 1):S4-S41

18. No authors listed (1997) Report of the expert committee on the diagnosis and classification of diabetes mellitus. Diabetes Care 20:1183-1197

19. Clark JD, Gebhart GF, Gonder JC, Keeling ME, Kohn DF (1997) Special report: the 1996 guide for the care and use of laboratory animals. ILAR J 38:41-48

20. Hori $\mathrm{H}, \mathrm{Gu} \mathrm{YJ}$, Nagata $\mathrm{N}$ et al (2001) Isolation, culture, and characterization of endocrine cells from 6-month-old porcine pancreas. Cell Transplant 10:459-464

21. Grill V, Bjorklund A (2001) Overstimulation and beta-cell function. Diabetes 50(Suppl 1):S122-S124

22. Birkeland KI, Torjesen PA, Eriksson J, Vaaler S, Groop L (1994) Hyperproinsulinemia of type II diabetes is not present before the development of hyperglycemia. Diabetes Care 17: 1307-1310

23. Ohagi S, Yoshida H, Nanjo K (1994) Analysis of the gene encoding human PC2, a prohormone processing enzyme. Nippon Rinsho 52:2544-2549 (article in Japanese)

24. Ouafik LH, Mattei MG, Giraud P, Oliver C, Eipper BA, Mains RE (1993) Localization of the gene encoding peptidylglycine alphaamidating monooxygenase (PAM) to human chromosome 5q145q21. Genomics 18:319-321 
25. Goodge KA, Hutton JC (2000) Translational regulation of proinsulin biosynthesis and proinsulin conversion in the pancreatic beta-cell. Semin Cell Dev Biol 11:235-242

26. Rouille Y, Westermark G, Martin SK, Steiner DF (1994) Proglucagon is processed to glucagon by prohormone convertase PC2 in alpha TC1-6 cells. Proc Natl Acad Sci U S A 91:3242-3246

27. Smeekens SP (1993) Processing of protein precursors by a novel family of subtilisin-related mammalian endoproteases. Biotechnology (NY) 11:182-186

28. Graw J, Loster J, Puk O et al (2005) Three novel Pax6 alleles in the mouse leading to the same small-eye phenotype caused by different consequences at target promoters. Invest Ophthalmol Vis Sci 46:4671-4683

29. Marzban L, Soukhatcheva G, Verchere CB (2005) Role of carboxypeptidase $\mathrm{E}$ in processing of pro-islet amyloid polypeptide in $\beta$-cells. Endocrinology 146:1808-1817

30. Jia ZM, Xu W, Yu L et al (2005) Effects of cyclosporin A on gene expression profiles of NIT-1 pancreatic beta cell line. Di Yi Jun Yi Da Xue Xue Bao [Academic Journal of the First Medical College of PLA] 25:853-857 (article in Chinese)

31. Pritchard LE, Turnbull AV, White A (2002) Pro-opiomelanocortin processing in the hypothalamus: impact on melanocortin signalling and obesity. J Endocrinol 172:411-421
32. Lloyd DJ, Bohan S, Gekakis N (2006) Obesity, hyperphagia and increased metabolic efficiency in Pc1 mutant mice. Hum Mol Genet 15:1884-1893

33. Jackson RS, Creemers JW, Farooqi IS et al (2003) Small-intestinal dysfunction accompanies the complex endocrinopathy of human proprotein convertase 1 deficiency. J Clin Invest 112:1550-1560

34. Zhu X, Zhou A, Dey A et al (2002) Disruption of PC1/3 expression in mice causes dwarfism and multiple neuroendocrine peptide processing defects. Proc Natl Acad Sci U S A 99:1029310298

35. Jackson RS, Creemers JW, Ohagi S et al (1997) Obesity and impaired prohormone processing associated with mutations in the human prohormone convertase 1 gene. Nat Genet 16:303-306

36. Hill ME, Asa SL, Drucker DJ (1999) Essential requirement for Pax6 in control of enteroendocrine proglucagon gene transcription. Mol Endocrinol 13:1474-1486

37. Cao X, Flock G, Choi C, Irwin DM, Drucker DJ (2003) Aberrant regulation of human intestinal proglucagon gene expression in the NCI-H716 cell line. Endocrinology 144:2025-2033

38. Yano H, Kitano N, Morimoto M, Polonsky KS, Imura H, Seino Y (1992) A novel point mutation in the human insulin gene giving rise to hyperproinsulinemia (proinsulin Kyoto). J Clin Invest 89:1902-1907 